\title{
SOME SECOND-ORDER THREE-POINT BOUNDARY VALUE PROBLEM FOR DISCRETE EQUATIONS ON THE HALF-LINE
}

\author{
YU TIAN AND WEIGAO GE
}

\begin{abstract}
In this paper, we study the existence of multiple positive solutions of boundary value problems for secondorder three-point discrete equations

$$
\left\{\begin{array}{l}
\Delta^{2} x(n-1)-p \Delta x(n-1)-q x(n-1)+f(n, x(n))=0, \quad n \in N_{0} \\
x(0)=\alpha x(l), \quad x(\infty)=0
\end{array}\right.
$$

The proofs are based on the fixed point theorem in Fréchet space (see [7]).
\end{abstract}

\section{Introduction}

Let $N^{+}=\{0,1,2, \ldots\}, N_{0}=\{1,2, \ldots\}, N(a, b)=\{a, a+1, \ldots, b\}, a, b \in N^{+}$. This paper is concerned with the existence of multiple positive solutions to the following boundary value problem

$$
\begin{gathered}
\Delta^{2} x(n-1)-p \Delta x(n-1)-q x(n-1)+f(n, x(n))=0, \quad n \in N_{0}, \\
x(0)=\alpha x(l), \quad x(\infty)=0,
\end{gathered}
$$

here $p, \alpha \geq 0, l \in N^{+}, p, q \in R$ and $q>0,1+p>q$.

Let $C\left(N^{+}, R\right)$ denote the class of maps $\omega$ and continuous on $N^{+}$(discrete topology), the topology being that of uniform convergent on $N(0, m)$ for every $m \in N_{0}$.

Definition 1.1. A function $x \in C\left(N^{+}, R\right)$ is said to be a positive solution of boundary value problem (1.1) (1.2), if $x(i) \geq 0, i \in N^{+}, x$ satisfies (1.2) and (1.1) for $i \in N_{0}$.

If $x \in C\left(N^{+}, R\right)$, then for every $m \in N_{0}$, we define the semi-norm

$$
|x|_{m}=\sup _{i \in N(0, m)}|x(i)|(1+l)^{-i}, \quad l \in[0, \infty) .
$$

Note $C\left(N^{+}, R\right)$ is a Fréchet space. Also $C(N(0, m))$ denotes the space of maps $\omega$ continuous on $N(0, m)$ (discrete topology) with norm $|x|_{m}$.

Received September 12, 2006; revised November 12, 2006.

2000 Mathematics Subject Classification. 34K10, 39A10.

Key words and phrases. Fréchet space, Green's function, half-line, second-order discrete equations.

Supported by grand 10371006 from National Natural Sciences Foundation of P.R. China and grant 20050007011 from Foundation for Ph.D. Specialities of Educational Department of P.R. China, Tianyuan Fund of Mathematics in China(10726038). 
In the current work, we give the associate Green's function and obtain the existence of multiple positive solutions for BVP (1.1) (1.2) by employing fixed point theorem in Fréchet space (see [7]).

The motivation for the present work stems from both practical and theoretical aspects. In fact, particular cases of the continuous boundary value problem (BVP) on the half-line occur naturally in the study of radially symmetric solutions of nonlinear elliptic equations, see [11, 16], and various physical phenomena [9, 15], such as unsteady flow of gas through a semiinfinite porous media, the theory of drain flows, plasma physics, in determining the electrical potential in an isolated neutral atom. In all this applications, it is frequent that only solutions that are positive are useful. Recently there have been investigations on the positive solutions of boundary value problem, see [1-8, 10, 12-14, 17-25]. Agarwal et al. in [3] have considered the following two-point boundary value problem

$$
\left\{\begin{array}{l}
\Delta^{2} x(k-1)+f(k, x(k))=0, \quad k \in N \backslash\{0\}, \\
x(0)=0, \quad x(\infty)=\gamma \in R .
\end{array}\right.
$$

By employing upper and lower solutions method, one positive solution is obtained. Liu [18], [19], Zima [25] studied the existence of positive solutions of second-order two-point boundary value problems for differential equations. However, no results were established for multiple point boundary value problems of discrete equations on the half-line. It is well known that the study of multiple point boundary value problems is very important. For finite interval, there have been many results, see $[8,20,21]$. So it is necessary that we discuss the existence of the positive solutions for the three-point boundary value problem on the half-line. The goal of this paper is to fill the gap in this area. Furthermore, the existence of multiple positive solutions is given. Our results extend and complete many results in the literatures, see $[1,2$, $3,4,5,10,14]$ and the references therein.

The paper is organized as follows: Section 2 presents the expression and some useful properties for Green's function to the problem

$$
-\Delta^{2} x(n-1)+p \Delta x(n-1)+q x(n-1)=0, x(0)=\alpha x(l), x(\infty)=0
$$

(see Lemma 2.1 and Lemma 2.2). In Section 3 we first state fixed point theorem in Fréchet space from [7], then applying the fixed point theorem the existence of one positive solution of BVP (1.1) (1.2) is established. In Section 4, the existence of multiple positive solutions is established.

\section{Related Lemmas}

For convenience, let

$$
a=\frac{p+\sqrt{p^{2}+r q}}{2}, \quad b=\frac{p-\sqrt{p^{2}+4 q}}{2} .
$$

It is easy to see that $a>0, b<0$. By $1+p>q$ we have $b+1>0$.

The following conditions will be used in this paper: 
(H1) $f: N^{+} \times R \rightarrow R$ is continuous with $f: N^{+} \times[0, \infty) \rightarrow[0, \infty), y \mapsto f(i, y)$ are nondecreasing for every $i \in N^{+}$;

(H2)

$$
\alpha(1+a)^{l}<1
$$

Lemma 2.1. Let $g \in C\left(N^{+}, R\right)$ and $\sum_{i=1}^{\infty} \frac{g(i)}{(1+a)^{i}}<\infty$. Then $x \in C^{2}\left(N^{+}, R\right)$ is a solution of the following boundary value problem

$$
\left\{\begin{array}{l}
\Delta^{2} x(n-1)-p \Delta x(n-1)-q x(n-1)+g(n)=0, \quad n \in N_{0}, \\
x(0)=\alpha x(l), \quad x(\infty)=0
\end{array}\right.
$$

if and only if $x \in C\left(N^{+}, R\right)$ is a solution of the following equation

$$
x(n)=\sum_{i=1}^{\infty} G(n, i) g(i)
$$

where

$$
\begin{aligned}
G(n, i) & =\frac{1}{\Gamma} \\
& \times \begin{cases}(1+b)^{n+i}\left[(1+b)^{-i}-(1+a)^{-i}\right], \quad i \leq n-i, & i \leq l-1, \\
(1+b)^{i}(1+a)^{n-i}\left[1-\alpha(1+b)^{l}\right]-(1+b)^{n+i}\left[(1+a)^{-i}-\alpha(1+b)^{l-i}\right], & n \leq i \leq l-1, \\
(1+b)^{n+i}\left\{(1+b)^{-i}\left[1-\alpha(1+b)^{l}\right]-(1+a)^{-i}\left[1-\alpha(1+a)^{l}\right]\right\}, & l \leq i \leq n-1, \\
\left(\frac{1+b}{1+a}\right)^{i}\left\{(1+a)^{n}\left[1-\alpha(1+b)^{l}\right]-(1+b)^{k}\left[1-\alpha(1+a)^{l}\right]\right\}, & i \geq n, i \geq l,\end{cases}
\end{aligned}
$$

$\Gamma=(a-b)\left[1-\alpha(1+b)^{l}\right]$.

Proof. Set

$$
u(n)=\Delta x(n)-a x(n)
$$

By the equation in (2.1) we have

$$
\begin{aligned}
\Delta u(n-1)-b u(n-1) & =\Delta^{2} x(n-1)-a \Delta x(n-1)-b[\Delta x(n-1)-a x(n-1)] \\
& =\Delta^{2} x(n-1)-(a+b) \Delta x(n-1)+a b x(n-1) \\
& =\Delta^{2} x(n-1)-p \Delta x(n-1)-q x(n-1)=-g(n), \quad n \in N_{0},
\end{aligned}
$$

i.e.

$$
u(n)-(1+b) u(n-1)=-g(n), \quad n \in N_{0} .
$$

Multiplying (2.4) by $\left(\frac{1}{1+b}\right)^{n}$ and summing on $N(1, k)$ we get

$$
\sum_{n=1}^{k}\left[u(n)\left(\frac{1}{1+b}\right)^{n}-u(n-1)\left(\frac{1}{1+b}\right)^{n-1}\right]=-\sum_{n=1}^{k}\left(\frac{1}{1+b}\right)^{n} g(n),
$$


i.e.

$$
u(k)=(1+b)^{k}\left[u(0)-\sum_{n=1}^{k}\left(\frac{1}{1+b}\right)^{n} g(n)\right] .
$$

Similarly, multiplying (2.3) by $\left(\frac{1}{1+a}\right)^{n}$ and summing on $N(1, k-1)$, we get

$$
x(k)\left(\frac{1}{1+a}\right)^{k-1}=x(1)+\sum_{n=1}^{k-1}\left(\frac{1}{1+a}\right)^{n} u(n)
$$

i.e.

$$
x(k)=(1+a)^{k-1} x(1)+(1+a)^{n-1} \sum_{n=1}^{k-1}\left(\frac{1}{1+a}\right)^{n} u(n) .
$$

By (2.5), (2.6) we get

$$
x(n)=(1+a)^{n-1} x(1)+(1+a)^{n-1} \sum_{n=1}^{n-1}\left(\frac{1+b}{1+a}\right)^{k}\left[u(0)-\sum_{i=1}^{k} \frac{g(i)}{(1+b)^{i}}\right] .
$$

From (2.3), (2.7) we have

$$
x(n)=\frac{1}{a-b}\left\{A(1+a)^{n}+B(1+b)^{n}-(1+a)^{n} \sum_{i=1}^{n-1}\left[\left(\frac{1+b}{a+a}\right)^{i}-\left(\frac{1+b}{1+a}\right)^{n}\right] g(i)\right\} .
$$

where $A=x(1)-(1+b) x(0), B=(1+a) x(0)-x(1)$.

Let $n=l$ in (2.8), we have

$$
x(l)=\frac{1}{a-b}\left\{A(1+a)^{l}+B(1+b)^{l}-(1+a)^{l} \sum_{i=1}^{l-1}\left[\left(\frac{1+b}{1+a}\right)^{i}-\left(\frac{1+b}{1+a}\right)^{l}\right] g(i)\right\} .
$$

By $x(0)=\alpha x(l)$ and (2.9) we have

$$
(a-b) x(0)=\alpha\left\{A(1+a)^{l}+B(1+b)^{l}-(1+a)^{l} \sum_{i=1}^{l-1}\left[\left(\frac{1+b}{1+a}\right)^{i}-\left(\frac{1+b}{1+a}\right)^{l}\right] g(i)\right\} .
$$

By boundary condition,

$$
\begin{aligned}
0 & =\lim _{n \rightarrow \infty} \frac{x(n)}{(1+a)^{n}}=\lim _{n \rightarrow \infty} \frac{1}{a-b}\left\{A+B\left(\frac{1+b}{1+a}\right)^{n}-\sum_{i=1}^{n-1}\left[\left(\frac{1+b}{1+a}\right)^{i}-\left(\frac{1+b}{1+a}\right)^{n}\right] g(i)\right\} \\
& =\frac{1}{a-b}\left\{A-\sum_{i=1}^{\infty}\left(\frac{1+b}{1+a}\right)^{i} g(i)+\lim _{n \rightarrow \infty}\left(\frac{1+b}{1+a}\right)^{n} \sum_{i=1}^{n-1} g(i)\right\} .
\end{aligned}
$$

If $\sum_{i=1}^{\infty} g(i)<\infty$, then $\lim _{n \rightarrow \infty}\left(\frac{1+b}{1+a}\right)^{n} \sum_{i=1}^{n-1} g(i)=0$.

If $\sum_{i=1}^{\infty} g(i)=\infty$, then by the rule of L'Hospital, we have

$$
\lim _{n \rightarrow \infty}\left(\frac{1+b}{1+a}\right)^{n} \sum_{i=1}^{n-1} g(i)=\lim _{n \rightarrow \infty} \frac{g(n)}{\left(\frac{1+a}{1+b}\right)^{n} \frac{a-b}{1+b}}=\lim _{n \rightarrow \infty} \frac{(1+b)^{n+1} g(n)}{(a-b)(1+a)^{n}}
$$




$$
\leq \lim _{n \rightarrow \infty} \frac{g(n)}{(a-b)(1+a)^{n}} .
$$

The condition $\sum_{i=1}^{\infty} \frac{g(i)}{(1+a)^{i}}<\infty$ means that $\lim _{n \rightarrow \infty} \frac{g(n)}{(1+a)^{n}}=0$. So

$$
\lim _{n \rightarrow \infty}\left(\frac{1+b}{1+a}\right)^{n} \sum_{i=1}^{n-1} g(i)=0
$$

Therefore (2.11) means that

$$
A=\sum_{i=1}^{\infty}\left(\frac{1+b}{1+a}\right)^{i} g(i)
$$

Since $(a-b) x(0)=A+B=\sum_{i=1}^{\infty}\left(\frac{1+b}{1+a}\right)^{i} g(i)+B$ and (2.10), we have

$$
\sum_{i=1}^{\infty}\left(\frac{1+b}{1+a}\right)^{i} g(i)+B=\alpha\left\{A(1+a)^{l}+B(1+b)^{l}-(1+a)^{l} \sum_{i=1}^{l-1}\left[\left(\frac{1+b}{1+a}\right)^{i}-\left(\frac{1+b}{1+a}\right)^{l}\right] g(i)\right\},
$$

thus

$$
\begin{aligned}
B=\frac{1}{1-\alpha(1+b)^{l}}\{ & {\left[\alpha(1+a)^{l}-1\right] \sum_{i=1}^{\infty}\left(\frac{1+b}{1+a}\right)^{i} g(i) } \\
& \left.-\alpha(1+a)^{l} \sum_{i=1}^{l-1}\left[\left(\frac{1+b}{1+a}\right)^{i}-\left(\frac{1+b}{1+a}\right)^{l}\right] g(i)\right\} .
\end{aligned}
$$

Thus substituting (2.12) and (2.13) into (2.8), we have

$$
\begin{aligned}
x(n)= & \frac{1}{a-b}\left\{\frac{(1+a)^{n}-\alpha(1+a)^{n}(1+b)^{l}+\alpha(1+b)^{n}(1+a)^{l}-(1+b)^{n}}{1-\alpha(1+b)^{l}} \sum_{i=1}^{\infty}\left(\frac{1+b}{1+a}\right)^{i} g(i)\right. \\
& -\frac{\alpha(1+a)^{l}(1+b)^{n}}{1-\alpha(1+b)^{l}} \sum_{i=1}^{l}\left[\left(\frac{1+b}{1+a}\right)^{i}-\left(\frac{1+b}{1+a}\right)^{l}\right] g(i) \\
& \left.-(1+a)^{n} \sum_{i=1}^{n}\left[\left(\frac{1+b}{1+a}\right)^{i}-\left(\frac{1+b}{1+a}\right)^{l}\right] g(i)\right\} .
\end{aligned}
$$

After a tedious computation we have (2.2) holds.

On the other hand, applying the difference operator directly to (2.2) gives (2.1).

Lemma 2.2. Suppose that $G(n, i)$ is given by Lemma 2.1 and the condition (H2) holds, then we have

(i) $G(n, i) \geq 0, n \in N^{+}, i \in N_{0}$.

(ii) $G(n, i)(1+a)^{-n} \leq G(i, i)(1+a)^{-i}, G(i, i) \leq \frac{1}{\Gamma}, n, i \in N_{0}$.

(iii) For any $l_{1}, l_{2} \in N_{0}, l_{2}>l_{1}$, there exists $\gamma=\min \left\{(1+b)^{l_{2}},(1+a)^{l_{1}}-(1+b)^{l_{1}}\right\}$ such that

$$
G(n, i) \geq \gamma G(i, i)(1+a)^{-i}, \quad n \in N\left(l_{1}, l_{2}\right) .
$$


Proof. It is clear that (i) holds. Now we show that (ii) holds. For the case $i \leq n-1, i \leq l-1$, we have

$$
(1+a)^{-n} G(n, i) \leq(1+a)^{-i} G(i, i)
$$

and

$$
G(i, i)=\frac{1}{\Gamma}(1+b)^{i}\left[1-(1+a)^{-i}(1+b)^{i}\right] \leq \frac{1}{\Gamma}(1+b)^{-i} \leq \frac{1}{\Gamma} .
$$

For the case $n \leq i \leq l-1$, we have

$$
\Gamma(1+a)^{-n} G(n, i)=\left(\frac{1+b}{1+a}\right)^{i}\left[1-\alpha(1+b)^{l}\right]-\frac{(1+b)^{n+i}}{(1+a)^{n}}\left[(1+a)^{-i}-\alpha(1+b)^{l-i}\right] .
$$

Notice the condition (H2) we have

$$
\begin{aligned}
(1+a)^{-i}-\alpha(1+b)^{l-i} & =(1+a)^{-i}\left[1-\alpha(1+a)^{i}(1+b)^{l-i}\right] \\
& \geq(1+a)^{-i}\left[1-\alpha(1+a)^{l}\right]>0
\end{aligned}
$$

So

$$
\begin{aligned}
\Gamma(1+a)^{-n} G(n, i) & \leq\left(\frac{1+b}{1+a}\right)^{i}\left[1-\alpha(1+b)^{l}\right]-\frac{(1+b)^{2 i}}{(1+a)^{i}}\left[(1+a)^{-i}-\alpha(1+b)^{l-i}\right] \\
& =\Gamma(1+a)^{-i} G(i, i) . \\
G(i, i)= & \frac{1}{\Gamma}\left\{(1+b)^{i}\left[1-\alpha(1+b)^{l}\right]-(1+b)^{2 i}\left[(1+a)^{-i}-\alpha(1+b)^{l-i}\right]\right\} \\
= & \frac{1}{\Gamma}(1+b)^{i}\left[1-\left(\frac{1+b}{1+a}\right)^{i}\right] \leq \frac{1}{\Gamma} .
\end{aligned}
$$

For the case $l \leq i \leq n-1$, we have

$$
\begin{aligned}
\Gamma(1+a)^{-n} G(n, i) & =\frac{(1+b)^{n+i}}{(1+a)^{n}}\left\{(1+b)^{-i}\left[1-\alpha(1+b)^{l}\right]-(1+a)^{-i}\left[1-\alpha(1+a)^{l}\right]\right\} \\
& =\left(\frac{1+b}{1+a}\right)^{n}\left[1-\alpha(1+b)^{l}\right]-\left(\frac{1+b}{1+a}\right)^{n+i}\left[1-\alpha(1+a)^{l}\right] \\
& =\left(\frac{1+b}{1+a}\right)^{n}\left\{1-\alpha(1+b)^{l}-\left(\frac{1+b}{1+a}\right)^{i}\left[1-\alpha(1+a)^{l}\right]\right\} .
\end{aligned}
$$

Since

$$
\begin{aligned}
1-\alpha(1+b)^{l}-\left(\frac{1+b}{1+a}\right)^{i}\left[1-\alpha(1+a)^{l}\right] & \geq 1-\alpha(1+b)^{l}-1+\alpha(1+a)^{l} \\
& =\alpha\left[(1+a)^{l}-(1+b)^{l}\right]>0
\end{aligned}
$$

we have

$$
\Gamma(1+a)^{-n} G(n, i) \leq\left(\frac{1+b}{1+a}\right)^{i}\left\{1-\alpha(1+b)^{l}-\left(\frac{1+b}{1+a}\right)^{i}\left[1-\alpha(1+a)^{l}\right]\right\}
$$




$$
\begin{gathered}
=\Gamma(1+a)^{-i} G(i, i) . \\
G(i, i)=\frac{1}{\Gamma}(1+b)^{i}\left\{1-\alpha(1+b)^{l}-\left(\frac{1+b}{1+a}\right)^{i}\left[1-\alpha(1+a)^{l}\right]\right\} \leq \frac{1}{\Gamma}(1+b)^{i} \leq \frac{1}{\Gamma} .
\end{gathered}
$$

For $i \geq n, i \geq l$, noticing $1+b>0$, we have

$$
\begin{aligned}
\frac{G(n, i)(1+a)^{-n}}{G(i, i)(1+a)^{-i}} & =\frac{(1+a)^{-n}\left(\frac{1+b}{1+a}\right)^{i}\left\{(1+a)^{n}\left[1-\alpha(1+b)^{l}\right]-(1+b)^{n}\left[1-\alpha(1+a)^{l}\right]\right\}}{(1+a)^{-i}\left(\frac{1+b}{1+a}\right)^{i}\left\{(1+a)^{i}\left[1-\alpha(1+b)^{l}\right]-(1+b)^{i}\left[1-\alpha(1+a)^{l}\right]\right\}} \\
& =\frac{1-\alpha(1+b)^{l}-\left(\frac{1+b}{1+a}\right)^{n}\left[1-\alpha(1+a)^{l}\right]}{1-\alpha(1+b)^{l}-\left(\frac{1+b}{1+a}\right)^{i}\left[1-\alpha(1+a)^{l}\right]} \\
& \leq 1 .
\end{aligned}
$$

So $(1+a)^{-n} G(n, i) \leq(1+a)^{-i} G(i, i)$.

$$
\begin{aligned}
G(i, i) & =\frac{1}{\Gamma}\left(\frac{1+b}{1+a}\right)^{i}\left\{(1+a)^{i}\left[1-\alpha(1+b)^{l}\right]-(1+b)^{i}\left[1-\alpha(1+a)^{l}\right]\right\} \\
& \leq \frac{1}{\Gamma}\left\{(1+b)^{i}\left[1-\alpha(1+b)^{l}\right]-\frac{(1+b)^{2 i}}{(1+a)^{i}}\left[1-\alpha(1+a)^{l}\right]\right\} \\
& \leq \frac{1}{\Gamma} .
\end{aligned}
$$

Finally we prove that (iii) holds.

Let $l_{1}, l_{2} \in N_{0}, l_{2}>l_{1}$.

For $i \leq n-1, i \leq l-1$, we have

$$
\begin{aligned}
\frac{G(n, i)}{G(i, i)(1+a)^{-i}} & =\frac{(1+b)^{n+i}\left[(1+b)^{-i}-(1+a)^{-i}\right]}{(1+b)^{2 i}\left[(1+b)^{-i}-(1+a)^{-i}\right](1+a)^{-i}} \\
& =\frac{(1+b)^{n}(1+a)^{i}}{(1+b)^{i}} \geq(1+b)^{l_{2}}, \quad n \in N\left(l_{1}, l_{2}\right)
\end{aligned}
$$

For $n \leq i \leq l-1$, we have

$$
\begin{aligned}
\frac{G(n, i)}{G(i, i)(1+a)^{-i}} & =\frac{(1+b)^{i}(1+a)^{n-i}\left[1-\alpha(1+b)^{l}\right]-(1+b)^{n+i}\left[(1+a)^{-i}-\alpha(1+b)^{l-i}\right]}{(1+a)^{-i}(1+b)^{i}\left[1-\alpha(1+b)^{l}\right]-(1+b)^{2 i}\left[(1+a)^{-i}-\alpha(1+b)^{l-i}\right](1+a)^{-i}} \\
& =\frac{(1+a)^{n-i}\left[1-\alpha(1+b)^{l}\right]-\frac{(1+b)^{n}}{(1+a)^{i}}\left[1-\alpha(1+b)^{l-i}(1+a)^{i}\right]}{(1+a)^{-i}\left[1-\alpha(1+b)^{l}\right]-\left(\frac{1+b}{1+a}\right)^{i}(1+a)^{-i}\left[1-\alpha(1+b)^{l-i}(1+a)^{i}\right]} \\
& \geq \frac{(1+a)^{l_{1}-i}\left[1-\alpha(1+b)^{l}\right]-\frac{(1+b)^{l_{1}}}{(1+a)^{i}}\left[1-\alpha(1+b)^{l-i}(1+a)^{i}\right]}{(1+a)^{-i}\left[1-\alpha(1+b)^{l}\right]} \\
& \geq(1+a)^{l_{1}}-(1+b)^{l_{1}}, \quad n \in N\left(l_{1}, l_{2}\right) .
\end{aligned}
$$


For $l \leq i \leq n-1$, we have

$$
\begin{aligned}
\frac{G(n, i)}{G(i, i)(1+a)^{-i}} & =\frac{(1+b)^{n+i}\left\{(1+b)^{-i}\left[1-\alpha(1+b)^{l}\right]-(1+a)^{-i}\left[1-\alpha(1+a)^{l^{l}}\right]\right\}}{(1+b)^{2 i}(1+a)^{-i}\left\{(1+b)^{-i}\left[1-\alpha(1+b)^{l}\right]-(1+a)^{-i}\left[1-\alpha(1+a)^{l}\right]\right\}} \\
& =(1+b)^{n-i}(1+a)^{i} \geq(1+b)^{l_{2}}, \quad n \in N\left(l_{1}, l_{2}\right) .
\end{aligned}
$$

For $i \geq n, i \geq l$, we have

$$
\begin{aligned}
\frac{G(n, i)}{G(i, i)(1+a)^{-i}} & =\frac{\left(\frac{1+b}{1+a}\right)^{i}\left\{(1+a)^{n}\left[1-\alpha(1+b)^{l}\right]-(1+b)^{n}\left[1-\alpha(1+a)^{l}\right]\right\}}{\left(\frac{1+b}{1+a}\right)^{i}\left\{(1+a)^{i}\left[1-\alpha(1+b)^{l}\right]-(1+b)^{i}\left[1-\alpha(1+a)^{l}\right]\right\}(1+a)^{-i}} \\
& \geq \frac{(1+a)^{n}-(1+b)^{n}-\alpha(1+a)^{n}(1+b)^{l}+\alpha(1+b)^{n}(1+a)^{l}}{1-\alpha(1+b)^{l}} \\
& =(1+a)^{n} \frac{(1+b)^{n}\left[1-\alpha(1+a)^{l}\right]}{1-\alpha(1+b)^{l}} \\
& \geq(1+a)^{n}-(1+b)^{n} \\
& \geq(1+a)^{l_{1}}-(1+b)^{l_{1}}, \quad n \in N\left(l_{1}, l_{2}\right) .
\end{aligned}
$$

Therefore, (iii) holds. 


\section{Existence Based on a Cone Compression and Expansion Theorem in Fréchet Space}

We first present the fixed point result from [7] that we will use in the Section 3 and Section 4. Let $E$ be a Fréchet space endowed with a family of semi-norms $\left\{|\cdot|_{n}: n \in N_{0}\right\}$ with

$$
|x|_{1} \leq|x|_{2} \leq \cdots, \quad \text { for all } x \in E .
$$

Also assume for each $n \in N_{0}$ that $\left(E_{n},|\cdot|_{n}\right)$ is a Banach space, and suppose

$$
E_{1} \supseteq E_{2} \supseteq \cdots
$$

with $E=\cap_{n=1}^{\infty} E_{n}$ and $|x|_{n} \leq|x|_{n+1}$ for all $x \in E_{n+1}$ (here $n \in N_{0}$ ). For each $n \in N_{0}$, let $C_{n}$ be a cone in $E_{n}$ and assume that $|\cdot|_{n}$ is increasing with respect to $C_{n}$. Also assume

$$
C_{1} \supseteq C_{2} \supseteq \cdots .
$$

For $\rho>0$ and $n \in N_{0}$, let

$$
U_{n, \rho}=\left\{x \in E_{n}:|x|_{n}<\rho\right\} \text { and } \Omega_{n, \rho}=U_{n, \rho} \cap C_{n} .
$$

Notice

$$
\partial_{C_{n}} \Omega_{n, \rho}=\partial_{E_{n}} U_{n, \rho} \cap C_{n} \text { and } \overline{\Omega_{n, \rho}}=\overline{U_{n, \rho}} \cap C_{n}
$$

(the first closure is with respect to $C_{n}$, whereas the second is with respect to $E_{n}$ ). In addition, notice since $|x|_{n} \leq|x|_{n+1}$ for all $x \in E_{n+1}$ that

$$
\Omega_{1, \rho} \supseteq \Omega_{2, \rho} \supseteq \cdots .
$$

Lemma 3.1.([6] Arzela-Ascoli Theorem for $C\left(N^{+}, R^{k}\right)$.) If $\Omega \subseteq C\left(N^{+}, R^{k}\right)$ is uniformly bounded on $N_{m}$ for each $m \in N^{+}$, then $\Omega$ is a relatively compact subset of $C\left(N^{+}, R^{k}\right)$.

We now present a general result which guarantees that the equation

$$
y=F y
$$

has a solution in $E$.

Theorem 3.2.(see [7].) Let $r_{1}>0, r_{2}>0, \bar{\gamma}>0$ be constants, $r=\min \left\{r_{1}, r_{2}\right\}$ and $R=$ $\max \left\{r_{i}, r_{2}\right\}$ with $\bar{\gamma}<r$. Suppose that the following conditions are satisfied:

$$
\begin{aligned}
& \text { for each } n \in N_{0}, F_{n}: \overline{U_{n, R}} \cap C_{n} \rightarrow C_{n} \text { is a continuous map; } \\
& \quad \text { for each } n \in N_{0},\left|F_{n} x\right|_{n} \geq|x|_{n} \text { for all } x \in \partial_{E_{n}} U_{n, r_{1}} \cap C_{n} ; \\
& \quad \text { for each } n \in N_{0},\left|F_{n} x\right|_{n} \leq|x|_{n} \text { for all } x \in \partial_{E_{n}} U_{n, r_{2}} \cap C_{n} ; \\
& \text { for each } n \in N_{0} \text {, the map } \mathscr{K}_{n}: \overline{U_{n, R}} \cap C_{n} \rightarrow C_{n} \text {, given by } \\
& \mathscr{K}_{n} y=\cup_{m=n}^{\infty} F_{m} y \text { (see Remark } 3.1 \text { ), is } k \text {-set contractive }(0<k<1 \text { ); }
\end{aligned}
$$




$$
\begin{aligned}
& \text { for every } k \in N_{0} \text { and any subsequence } A \subseteq\{k, k+1, \cdots\} \\
& \text { if } x \in C_{n}, n \in A \text {, is such that } R \geq|x|_{n} \geq r \text { then }|x|_{k} \geq \bar{\gamma}
\end{aligned}
$$

and

if there exists $a \omega \in E$ and a sequence $\left\{y_{n}\right\}_{n \in N_{0}}$ with $y_{n} \in\left(\overline{U_{n, R}} \backslash U_{n, r}\right) \cap C_{n}$ and $y_{n}=F_{n} y_{n}$ in $E_{n}$ such that for every $k \in N_{0}$, there exists a subsequence $S \subseteq\{k+1, k+2, \cdots\}$ of $N_{0}$ with $y_{n} \rightarrow \omega$ in $E_{k}$ as $n \rightarrow \infty$ in $S$, then $\omega=F_{\omega}$ in $E$.

Then (3.1) has a solution $y_{1} \in E$ with $y_{1} \in \cap_{n=1}^{\infty}\left(\left(\overline{U_{n, R}} \backslash U_{n, \bar{\gamma}}\right) \cap C_{n}\right)$.

Remark 3.1. The definition of $\mathscr{K}_{n}$ in (3.5) is as follows. If $y \in \overline{U_{n, R}} \cap C_{n}$ and $y \notin \overline{U_{n+1, R}} \cap$ $C_{n+1}$, then $\mathscr{K}_{n} y=F_{n} y$, whereas if $y \in \overline{U_{n+1, R}} \cap C_{n+1}$ and $y \notin \overline{U_{n+2, R}} \cap C_{n+2}$, then $\mathscr{K}_{n} y=F_{n} y \cup$ $F_{n+1} y$, and so on.

Theorem 3.3. Besides the conditions (H1) (H2) hold, we assume that the following conditions are satisfied:

(H3) there exist $R>r>o$, and $l_{2}>l_{1}>0, l_{1}, l_{2} \in N^{+}$such that

$$
\begin{gathered}
\sum_{j=1}^{\infty} \frac{1}{\Gamma}(1+a)^{-j} f\left(j, R(1+a)^{j}\right) \leq R ; \\
\gamma(1+a)^{-l_{2}} \sum_{j=l_{1}}^{l_{2}} G(j, j) f(j, \gamma r)(1+a)^{-j} \geq r
\end{gathered}
$$

hold. Then Problem (1.1) (1.2) has at least one positive solution $x \in C\left(N^{+}, R\right)$ with $x(i) \geq 0$ for $i \in N^{+}$and with

$$
\gamma r(1+a)^{-l_{2}} \leq \sup _{i \in N^{+}}|x(i)|(1+a)^{-i} \leq R, \quad x(i) \geq \gamma^{2} r(1+a)^{-l_{2}} \text { for each } i \in N\left(l_{1}, l_{2}\right) .
$$

Proof. Choose $\left\{b_{n}\right\} \subset N_{0}$ an increasing sequence such that $b_{1}=b \geq 1$ and $b_{n} \rightarrow \infty$ as $n \rightarrow \infty$. We endow $C\left(N^{+}, R\right)$ with a family of semi-norms $\left\{|\cdot|_{n}\right\}_{n \in N}$, defined as follows:

$$
|x|_{n}=\sup _{i \in N\left(0, b_{n}\right)}|x(i)|(1+a)^{-i} .
$$

We denote $E_{n}=C\left(N\left(0, b_{n}\right), R\right)$ and $C_{n}=\left\{x \in E_{n} \mid x(i) \geq 0\right.$ for $i \in N\left(0, b_{n}\right)$, and $\min _{i \in N\left(l_{1}, l_{2}\right)} x(i) \geq$ $\left.\gamma|x|_{n}\right\}$. Clearly $\left(E_{n},|\cdot|_{n}\right)$ is a Banach space.

Let $n \in N_{0}$. Define

$$
F_{n} x(i)=\sum_{j=1}^{b_{n}} G(i, j) f(j, x(j))
$$

here $x \in C\left(N,\left(1, b_{n}\right)\right)$. 
For $\rho=r, R$, let

$$
U_{n, \rho}=\left\{x \in C\left(0, b_{n}\right):|x|_{n}<\rho\right\} .
$$

We know from [6] that $F_{n}: \overline{U_{n, R}} \cap C_{n} \rightarrow E_{n}$ is continuous for each $n \in N_{0}$.

$$
\text { We claim that } F_{n}: \overline{U_{n, R}} \cap C_{n} \rightarrow C_{n} \text { for each } n \in N_{0} \text {. }
$$

If (3.11) is true, then of course (3.2) is true for $n \in N_{0}$. To see (3.11), fix $n \in N_{0}$ and take $x \in$ $\overline{U_{n, R}} \cap C_{n}$. Using (3.10) and inequality (ii) we obtain

$$
\begin{aligned}
\sup _{i \in N\left(0, b_{n}\right)}\left|F_{n} x(i)\right|(1+a)^{-i} & =\sup _{i \in N\left(0, b_{n}\right)} \sum_{j=1}^{b_{n}} G(i, j) f(j, x(j))(1+a)^{-i} \\
& \leq \sum_{j=1}^{b_{n}} G(j, j) f(j, x(j))(1+a)^{-j} .
\end{aligned}
$$

On the other hand, using inequality (iii) we have

$$
\begin{aligned}
\min _{i \in N\left(l_{1}, l_{2}\right)} F_{n} x(i) & =\min _{i \in N\left(l_{1}, l_{2}\right)} \sum_{j=1}^{b_{n}} G(i, j) f(j, x(j)) \\
& \geq \gamma \sum_{j=1}^{b_{n}} G(j, j)(1+a)^{-j} f(j, x(j)) \\
& \leq \gamma \sup _{i \in N\left(0, b_{n}\right)}\left|F_{n} x(i)\right|(1+a)^{-i}=\gamma\left|F_{n} x\right|_{n} .
\end{aligned}
$$

By $G(i, j) \geq 0,(i, j) \in N^{+} \times N_{0}$ and (H1) we have $F_{n} x(i) \geq 0$ for $i \in N\left(0, b_{n}\right)$. Thus (3.11) (also (3.2) with $n \in N_{0}$ ) is true.

Next we show (3.3) (with $r_{1}=r$ ) with $n \in N_{0}$. Let $x \in \partial_{E_{n}} U_{n, r} \cap C_{n}$. Note $|x|_{n}=r$ and $x(i) \geq \gamma|x|_{n}=\gamma r$ for $i \in N\left(l_{1}, l_{2}\right)$. Noticing (H1) (3.9) (3.10) and inequality (iii) we have

$$
\begin{aligned}
\left|F_{n} x(i)\right|_{n} & =\sup _{i \in N\left(0, b_{n}\right)} \sum_{j=1}^{b_{n}} G(i, j) f(j, x(j))(1+a)^{-i} \\
& \geq \inf _{i \in N\left(l_{1}, l_{2}\right)} \sum_{j=l_{1}}^{l_{2}} G(i, j) f(j, x(j))(1+a)^{-i} \\
& \geq \inf _{i \in N\left(l_{1}, l_{2}\right)} \gamma \sum_{j=l_{1}}^{l_{2}} G(j, j) f(j, \gamma r)(1+a)^{-i-j} \\
& =\gamma(1+a)^{-l_{2}} \sum_{j=l_{1}}^{l_{2}} G(j, j) f(j, \gamma r)(1+a)^{-j} \\
& \geq r=|x|_{n} .
\end{aligned}
$$

So (3.3) (with $r_{1}=r$ ) is true for $n \in N_{0}$. 
To see (3.4) (with $r_{2}=R$ ) with $n \in N_{0}$ holds. Let $n \in N_{0}$ and $x \in \partial_{E_{n}} U_{n, R} \cap C_{n}$, so $\sup _{i \in N^{+}}|x(i)|(1+$ $a)^{-i}=R$, i.e. $x(i) \leq R(1+a)^{i}$ for $i \in N\left(0, b_{n}\right)$. Then using (3.8) and inequality (ii), we have

$$
\begin{aligned}
\left|F_{n} x(i)\right|(1+a)^{-i} & =\sum_{j=1}^{b_{n}} G(i, j) f(j, x(j))(1+a)^{-i} \\
& \leq \sum_{j=1}^{b_{n}} G(j, j) f(j, x(j))(1+a)^{-j} \\
& <\sum_{j=1}^{\infty} \frac{1}{\Gamma}(1+a)^{-j} f\left(j, R(1+a)^{j}\right) \leq R, \quad \text { for } \quad i \in N\left(0, b_{n}\right),
\end{aligned}
$$

which yields

$$
\left|F_{n} x\right|_{n} \leq R=|x|_{n} .
$$

Thus (3.4) (with $r_{2}=R$ ) holds for $n \in N_{0}$.

Fix $n \in N_{0}$, we will show $\mathscr{K}_{n}: \overline{U_{n, R}} \cap C_{n} \rightarrow C_{n}$, given by $\mathscr{K}_{n} x=\bigcup_{m=n}^{\infty} F_{m} x$ is compact. Let $x \in \overline{U_{n, R}} \cap C_{n}$. Without loss of generality, assume there exists $l \in\{0,1, \ldots\}$ with $x \in \overline{U_{n+l, R}} \cap C_{n+l}$ and $x \notin \overline{U_{n+l+1, R}} \cap C_{n+l+1}$. Then by definition (see Remark 3.1) $\mathscr{K}_{n} x=\bigcup_{m=n}^{n+l} F_{m} x$. Since $x \in$ $\overline{U_{n+l, R}} \cap C_{n+l}, x(j) \leq R(1+a)^{j}, j \in N^{+}$. By (H1) (ii) (3.8), we have for $m \in N(0, l), i \in N\left(0, b_{n}\right)$ that

$$
\begin{aligned}
\left|F_{n+m} x(i)\right|(1+a)^{-i} & =\sum_{j=1}^{b_{n+m}} G(i, j) f(j, x(j))(1+a)^{-i} \\
& \leq \sum_{j=1}^{b_{n+m}} G(j, j) f(j, x(j))(1+a)^{-j} \\
& \leq \sum_{j=1}^{\infty} \frac{1}{\Gamma}(1+a)^{-j} f\left(j, R(1+a)^{j}\right) \leq R,
\end{aligned}
$$

and so $M=\left\{y|y(i)=| F_{n+m} x(i) \mid(1+a)^{-i}, x \in \overline{U_{n+l, R}} \cap C_{n}\right\} \subseteq C\left(N\left(0, b_{n}\right)\right)$ is uniformly bounded according to the maximum norm. According to Lemma 3.1 (Arzelá-Ascoli theorem), $M$ is relatively compact, i.e. there exists a sequence $\left\{y_{k}\right\} \subseteq M$ and $y_{0} \in C\left(N\left(0, b_{n}\right)\right)$ such that $\lim _{k \rightarrow \infty} \max _{i \in N\left(0, b_{n}\right)} \mid y_{k}(i)-$ $y_{0}(i) \mid=0$. So

$$
\lim _{k \rightarrow \infty} \max _{i \in N\left(0, b_{n}\right)}\left|F_{n+m} x_{k}(i)(1+a)^{-i}-F_{n+m} x_{0}(i)(1+a)^{-i}\right|=0,
$$

which means $\lim _{k \rightarrow \infty}\left|F_{n+m} x_{k}-F_{n+m} x_{0}\right|=0$. Thus $M^{\prime}=\left\{z \mid z(i)=F_{n+m} x(i), x \in \overline{U_{n+l, R}} \cap C_{n}\right\}$ is relatively compact in $C_{n}$, and $\mathscr{K}_{n}: \overline{U_{n, R}} \cap C_{n} \rightarrow C_{n}$ is compact, so (3.5) holds.

We next will prove that (3.6) holds for $n \in N_{0}$ and $\bar{\gamma}=\gamma r(1+a)^{-l_{2}}$. To see this, fix $k \in N_{0}$ and take any subsequence $A \subseteq\{k, k+1, \cdots\}$ if $x \in C\left(0, b_{n}\right), n \in A$ is such that $r \leq|x|_{n} \leq R$, then $x(i) \geq \gamma|x|_{n} \geq \gamma r$ for $i \in N\left(l_{1}, l_{2}\right)$, and so $\min _{i \in N\left(l_{1}, l_{2}\right)} x(i) \geq \gamma r$. Thus

$$
|x|_{k}=\sup _{i \in N\left(0, b_{k}\right)}|x(i)|(1+a)^{-i} \geq \sup _{i \in N\left(l_{1}, l_{2}\right)}|x(i)|(1+a)^{-i} \geq \gamma r(1+a)^{-l_{2}},
$$


so (3.6) holds for $n \in N_{0}$.

It remains to show that (3.7) is satisfied. Suppose there exists $\omega \in C\left(N_{0}, R\right)$ and a sequence $\left\{x_{n}\right\}_{n \in N_{0}}$ with $x_{n} \in \overline{U_{n, R}} \cap C_{n}$ and $x_{n}(i)=F_{n} x_{n}(i), i \in N\left(0, b_{n}\right)$ such that for every $k \in N_{0}$, there exists a subsequence $S \subseteq\{k+1, k+2, \cdots\}$ of $N_{0}$ with $x_{n} \rightarrow \omega$ in $C\left(N\left(0, b_{k}\right), R\right)$ as $n \rightarrow \infty$ in $S$. If we show

$$
\omega(i)=\sum_{j=1}^{\infty} G(i, j) f(j, \omega(j)) \text { for } i \in N^{+}
$$

then (3.7) holds.

Fix $i \in N^{+}$. Consider $k \geq i$ and $n \in S$ (as described above). Then $x_{n}(i)=F_{n} x_{n}(i), i \in$ $N\left(0, b_{n}\right)$ for $n \in S$, and so

$$
x_{n}(i)-\sum_{j=1}^{b_{k}} G(i, j) f\left(j, x_{n}(j)\right)=\sum_{j=b_{k}+1}^{b_{n}} G(i, j) f\left(j, x_{n}(j)\right) .
$$

From $x_{n} \in \overline{U_{n, R}} \cap C_{n}$, we have $x_{n}(i) \leq R(1+a)^{i}$. Now by (3.8) and inequality (ii) we have

$$
\begin{aligned}
(1+a)^{-i}\left|\sum_{j=b_{k+1}}^{b_{n}} G(i, j) f\left(j, x_{n}(j)\right)\right| & \leq \sum_{j=b_{k+1}}^{\infty} G(i, j) f\left(j, x_{n}(j)\right)(1+a)^{-i} \\
& \leq \sum_{j=b_{k+1}}^{\infty} G(j, j)(1+a)^{-j} f\left(j, R(1+a)^{j}\right) .
\end{aligned}
$$

So

$$
(1+a)^{-i}\left|x_{n}(i)-\sum_{j=1}^{b_{k}} G(i, j) f\left(j, x_{n}(j)\right)\right| \leq \sum_{j=b_{k+1}}^{\infty} G(j, j)(1+a)^{-j} f\left(j, R(1+a)^{j}\right)<R .
$$

Since $x_{n} \rightarrow \omega$ in $C\left(N\left(0, b_{k}\right), R\right)$ as $n \rightarrow \infty$ and (H1), let $n \rightarrow \infty$ in $C\left(N\left(0, b_{k}\right), R\right)$ through $S$ to obtain

$$
(1+a)^{-i}\left|\omega(i)-\sum_{j=1}^{b_{k}} G(i, j) f(j, \omega(j))\right| \leq \sum_{j=b_{k+1}}^{\infty} G(j, j)(1+a)^{-j} f\left(j, R(1+a)^{j}\right) .
$$

Finally let $k \rightarrow \infty$, we conclude by (3.8)

$$
(1+a)^{-i}\left|\omega(i)-\sum_{j=1}^{\infty} G(i, j) f(j, \omega(j))\right|=0,
$$

i.e.

$$
\omega(i)=\sum_{j=1}^{\infty} G(i, j) f(j, \omega(j))
$$

So (3.7) holds. Now apply Theorem $3.2 x(i)=\sum_{j=1}^{\infty} G(i, j) f(j, x(j))$ has a solution $x \in C\left(N^{+}, R\right)$ with $x(i) \geq 0$ for $i \in N^{+}$and

$$
\gamma r(1+a)^{-l_{2}} \leq \sup _{i \in N^{+}}|x(i)|(1+a)^{-i} \leq R, \quad x(i) \geq \gamma^{2} r(1+a)^{-l_{2}} \text { for each } i \in N\left(l_{1}, l_{2}\right) .
$$


By (3.8) we have

$$
\sum_{i=1}^{\infty}(1+a)^{-i} f(i, x(i)) \leq \sum_{i=1}^{\infty}(1+a)^{-i} f\left(i, R(1+a)^{i}\right)<\Gamma R<\infty .
$$

Thus by Lemma 2.1, $x$ is a positive solution of Problem (1.1) (1.2).

Theorem 3.4. Besides the conditions (H1) (H2) hold, we assume that there exist $l_{1}, l_{2} \in N_{0}$ and $l_{1}<l_{2}$ such that

$$
\begin{gathered}
\sup _{c \in N^{+}} \frac{c}{\sum_{j=1}^{\infty} \frac{1}{\Gamma}(1+a)^{-j} f\left(j, c(1+a)^{j}\right)}>1 \\
\lim _{r \rightarrow 0} \sum_{i=l_{1}}^{l_{2}} \frac{G(i, i) f(i, \gamma r)}{r(1+a)^{i}}=\infty .
\end{gathered}
$$

Then Problem (1.1) (1.2) has at least one positive solution.

Proof. By (3.13), choose an $R>0$ such that

$$
\frac{R}{\sum_{j=1}^{\infty} \frac{1}{\Gamma}(1+a)^{-j} f\left(j, R(1+a)^{j}\right)}>1,
$$

which implies (3.8). From (3.14), there exists an $r<R$ such that

$$
\sum_{i=l_{1}}^{l_{2}} \frac{G(i, i) f(i, \gamma r)}{(1+a)^{i} r}>\frac{(1+a)^{l_{2}}}{\gamma}
$$

which implies (3.9). So the conditions in Theorem 3.3 are satisfied. Applying Theorem 3.3, Problem (1.1) (1.2) has a positive solution.

\section{Existence of Multiple Positive Solutions}

Theorem 4.1. Suppose that the conditions in Theorem 3.4 hold. Furthermore, the following conditions hold

$$
\begin{gathered}
\lim _{r \rightarrow \infty} \sum_{i=l_{1}}^{l_{2}} \frac{G(i, i) f(i, \gamma r)}{r(1+a)^{i}}=\infty, \\
\sum_{j=1}^{\infty} \frac{f\left(i, k(1+a)^{i}\right)}{\Gamma(1+a)^{i}}<\infty, \quad \text { for any } k>0 .
\end{gathered}
$$

Then Problem (1.1) (1.2) has at least two positive solutions.

Proof. Choose $\left\{b_{n}\right\} \subset N_{0}$ an increasing sequence such that $b_{1}=b \geq 1$ and $b_{n} \rightarrow \infty$. We endow $C\left(N^{+}, R\right)$ with a family of semi-norms $\left\{|\cdot|_{n}\right\}_{n \in N}$, defined as follows:

$$
|x|_{n}=\sup _{i \in N\left(0, b_{n}\right)}|x(i)|(1+a)^{-i}
$$


We denote $E_{n}=C\left(N\left(0, b_{n}\right), R\right)$ and $C_{n}=\left\{x \in E_{n} \mid x(i) \geq 0\right.$ for $i \in N\left(0, b_{n}\right)$, and $\min _{i \in N\left(l_{1}, l_{2}\right)} x(i) \geq$ $\left.\gamma|x|_{n}\right\}$. Clearly $\left(E_{n},|\cdot|_{n}\right)$ is a Banach space.

Let $n \in N_{0}$. Define

$$
F_{n} x(i)=\sum_{j=1}^{b_{n}} G(i, j) f(j, x(j))
$$

here $x \in C\left(N\left(1, b_{n}\right)\right)$.

Now from condition (3.13) choose an $R$ such that

$$
\frac{R}{\sum_{j=1}^{\infty} \Gamma^{-1}(1+a)^{-j} f\left(j, R(1+a)^{j}\right)}>1 .
$$

Suppose $N^{*}=\frac{(1+a)^{l_{2}}}{\gamma}$. From (3.14) there exists $r \leq R$ such that

$$
\sum_{i=l_{1}}^{l_{2}} \frac{G(i, i) f(i, \gamma x)}{x(1+a)^{i}} \geq N^{*}=\frac{(1+a)^{l_{2}}}{\gamma}, \quad x \leq r
$$

From (4.1) there exists $R^{\prime}>R$ such that

$$
\sum_{i=l_{1}}^{l_{2}} \frac{G(i, i) f(i, \gamma x)}{x(1+a)^{i}} \geq \frac{(1+a)^{l_{2}}}{\gamma}, \quad x \geq R^{\prime} .
$$

Now we define for $\rho=r, R, R^{\prime}$,

$$
U_{n, \rho}=\left\{x \in C\left(0, b_{n}\right):|x|_{n}<\rho\right\} .
$$

First by Theorem 3.3 condition (H1) (4.4) (4.5) guarantee that there exists one positive solution $x_{1} \in C\left(N^{+}\right)$such that $x_{1}(i) \geq 0, i \in N^{+}$and

$$
\gamma r(1+a)^{-l_{2}} \leq \sup _{i \in N^{+}}\left|x_{1}(i)\right|(1+a)^{-i} \leq R^{\prime}, \quad x_{1}(i) \geq \gamma^{2} r(1+a)^{-l_{2}} \text { for each } n \in N\left(l_{1}, l_{2}\right)
$$

We next will prove that there exists another positive solution $x_{2} \in C\left(N^{+}\right)$such that $x_{2}(i) \geq$ $0, i \in N^{+}$and

$$
\gamma R(1+a)^{-l_{2}} \leq \sup _{i \in N^{+}}\left|x_{2}(i)\right|(1+a)^{-i} \leq R^{\prime}, \quad x_{2}(i) \geq \gamma^{2} R(1+a)^{-l_{2}} \text { for each } n \in N\left(l_{1}, l_{2}\right) .
$$

The proof is based on Theorem 3.2. First similar to Theorem 3.3 we get for each $n \in N_{0}, F_{n}$ : $\overline{U_{n, R^{\prime}}} \cap C_{n} \rightarrow C_{n}$ is a continuous map, that is to say, the condition (3.2) in Theorem 3.2 holds.

The following two claims hold:

Claim 1. $\left|F_{n} x\right|_{n} \geq|x|_{n}, x \in \partial_{E_{n}} U_{n, R^{\prime}} \cap C_{n}$. 
So $x(i) \geq \gamma|x|_{n}=\gamma R^{\prime}$ for $i \in N\left(l_{1}, l_{2}\right)$. By (4.6) and inequality (iii) we have

$$
\begin{aligned}
\left|F_{n} x(i)\right|(1+a)^{-i} & =\sup _{i \in N\left(0, b_{n}\right)} \sum_{j=1}^{b_{n}} G(i, j) f(j, x(j))(1+a)^{-i} \\
& \geq \gamma \sup _{i \in N\left(l_{1}, l_{2}\right)} \sum_{j=l_{1}}^{l_{2}} G(j, j)(1+a)^{-i-j} f\left(j, \gamma R^{\prime}\right) \\
& =\gamma(1+a)^{-l_{2}} \sum_{j=l_{1}}^{l_{2}} G(j, j) f\left(j, \gamma R^{\prime}\right)(1+a)^{-j} \\
& \geq R^{\prime}=|x|_{n} .
\end{aligned}
$$

Thus (3.3) holds for $r_{1}=R^{\prime}$.

Claim 2. $\left|F_{n} x\right|_{n} \leq|x|_{n}, x \in \partial_{E_{n}} U_{n, R} \cap C_{n}$.

Fix $n \in N_{0}$ and let $x \in \partial_{E_{n}} U_{n, R} \cap C_{n}$. So $x(i) \leq R(1+a)^{i}, i \in N^{+}$. By (4.4) and inequality (ii) we have

$$
\begin{aligned}
\left|F_{n} x(i)\right|(1+a)^{-i} & =\sum_{j=1}^{b_{n}} G(i, j) f(j, x(j))(1+a)^{-i} \\
& \leq \sum_{j=1}^{b_{n}} G(j, j) f(j, x(j))(1+a)^{-j} \\
& \leq \sum_{j=1}^{\infty} G(j, j) f\left(j, R(1+a)^{j}\right)(1+a)^{-j} \\
& \leq \sum_{j=1}^{\infty} \frac{f\left(j, R(1+a)^{j}\right)}{\Gamma(1+a)^{j}} \leq R .
\end{aligned}
$$

Thus (3.4) holds for $r_{2}=R$.

We next will show that (3.5) holds. For this, fix $n \in N_{0}$, we will show $\mathscr{K}_{n}: \overline{U_{n, R^{\prime}}} \cap C_{n} \rightarrow C_{n}$ given by $\mathscr{K}_{n} x=\bigcup_{m=n}^{\infty} F_{m} x$ is compact. Let $x \in \overline{U_{n, R^{\prime}}} \cap C_{n}$. Without loss of generality, assume there exists $l \in\{0,1, \ldots\}$ with $x \in \overline{U_{n+l, R^{\prime}}} \cap C_{n+l}$ and $x \notin \overline{U_{n+l+1, R^{\prime}}} \cap C_{n+l+1}$. Then by definition (see Remark 3.1) $\mathscr{K}_{n} x=\bigcup_{m=n}^{n+l} F_{m} x$. Since $x \in \overline{U_{n+l, R^{\prime}}} \cap C_{n+l}, x(j) \leq R^{\prime}(1+a)^{j}, j \in N^{+}$. By (ii) (4.2) we have for $m \in N(0, l), i \in N\left(0, b_{n}\right)$ that

$$
\begin{aligned}
\left|F_{n+m} x(i)\right|(1+a)^{-i} & =\sum_{j=1}^{b_{n+m}} G(i, j) f(j, x(j))(1+a)^{-i} \\
& \leq \sum_{j=1}^{b_{n+m}} G(j, j) f(j, x(j))(1+a)^{-j} \\
& \leq \sum_{j=1}^{\infty} \frac{1}{\Gamma}(1+a)^{-j} f\left(j, R^{\prime}(1+a)^{j}\right)<\infty
\end{aligned}
$$


So $\left|\mathscr{K}_{n} x\right|_{n}<\infty$. Similar to the process of Theorem 3.2 we have $\mathscr{K}_{n}: \overline{U_{n, R^{\prime}}} \cap C_{n} \rightarrow C_{n}$ is compact, so (3.5) holds.

Next we will prove (3.6) holds for $n \in N_{0}$ for $\bar{\gamma}=\gamma R(1+a)^{-l_{2}}$. To see this, fix $k \in N_{0}$ and take any subsequence $A \subseteq\{k, k+1, \cdots\}$ if $x \in C\left(0, b_{n}\right), n \in A$ is such that $R \leq|x|_{n} \leq R^{\prime}$, then $x(i) \geq \gamma|x|_{n} \geq \gamma R$ for $i \in N\left(l_{1}, l_{2}\right)$, and so $\inf _{i \in N\left(l_{1}, l_{2}\right)} x(i) \geq \gamma R$. Thus

$$
|x|_{k}=\sup _{i \in N\left(0, b_{k}\right)}|x(i)|(1+a)^{-i} \geq \inf _{i \in N\left(l_{1}, l_{2}\right)}|x(i)|(1+a)^{-i} \geq \gamma R(1+a)^{-l_{2}},
$$

so (3.6) holds for $n \in N_{0}$.

Similar to the proof in Theorem 3.3 we have (3.7) holds. Applying Theorem $3.2 \times(i)=$ $\sum_{j=1}^{\infty} G(i, j) f(j, x(j))$ has a positive solution $x_{2}$ such that

$$
\gamma R(1+a)^{-l_{2}} \leq \sup _{i \in N^{+}}\left|x_{2}(i)\right|(1+a)^{-i} \leq R^{\prime}, \quad x_{2}(i) \geq \gamma^{2} R(1+a)^{-l_{2}} \text { for each } n \in N\left(l_{1}, l_{2}\right) .
$$

By Lemma 2.1. we see that $x_{1}, x_{2}$ are two positive solutions of BVP (1.1) (1.2).

Remark 4.1. For $l=1, \alpha=\frac{\beta_{2}}{\beta_{1}+\beta_{2}}$, BVP (1.1) (1.2) is reduced to (1.1) and

$$
\beta_{1} x(0)-\beta_{2} \Delta x(0)=0 .
$$

where $\beta_{i} \geq 0, i=1,2, \beta_{1}^{2}+\beta_{2}^{2} \neq 0$, which has been studied in [23]. Our results in present paper generalize those in [23].

\section{References}

[1] R. P. Agarwal, Difference Equation and Inequalities, Marcel Dekker, New York, 1992.

[2] R. P. Agarwal, D. O'Regan and P. J. Y. Wong, Positive Solutions of Differential, Difference and Integral Equations, Kluwer Academic, Dordrecht, 1999.

[3] R. P. Agarwal and D. O’Regan, Infinite Interval Problems for Differential, Difference and Integral Equations, Kluwer Academic Publishes, Dordrecht/Boston/London, 2001.

[4] R. P. Agarwal and D. O'Regan, Boundary value problems for discrete equations, Appl. Math. Lett. 10(1997), 83-89.

[5] R. P. Agarwal and D. O'Regan, A fixed point approach for nonlinear discrete boundary value problems, Comput. Math. Appl. 36(1998), 115-121.

[6] R. P. Agarwal and D. O'Regan, Existence and approximation of solutions of nonlinear discrete systems on infinite intervals, Math. Meth. Appl. Sci. 22(1999), 91-99.

[7] R. P. Agarwal and D. O'Regan, Cone compression and expansion fixed point theorems in Fréchet spaces with application, J. Differential Equations 171(2001), 412-429.

[8] R. P. Agarwal, H. B. Thompson and C. C. Tisdell, Three-point boundary value problems for secondorder discrete equations, Comput. Math. Appl. 45(2003), 1429-1435.

[9] D. Aronson, M. G. Crandall and L. A. Peletier, Stabilization of solutions of a degenerate nonlinear diffusion problem, Nonlinear Anal. 6(1982), 1001-1022. 
[10] R. I. Avery and A. C. Peterson, Multiple positive solutions of a discrete second order conjugate problem, Panamer. Math. J. (1998), 1-12.

[11] J. V. Baxley, Existence and uniqueness of nonlinear boundary value problems on infinite intervals, J. Math. Anal. Appl. 147(1990), 127-133.

[12] K. L. Boey and P. J. Y. Wong, Two-point right focal eigenvalue problems on time scales, Appl. Math. Comput. 167(2005), 1281-1303.

[13] K. L. Boey and P. J. Y. Wong, Existence of triple positive solutions of two-point right focal boundary value problems on time scales, Comput. Math. Appl. 50(2005), 1603-1620.

[14] J. Henderson and H. B. Thompson, Existence of multiple solutions for second-order discrete boundary value problems, Comput. Math. Appl. 43(2002), 1239-1248.

[15] G. Iffland, Positive solutions of a problem Emden-Fowler type with a type free boundary, SIAM J. Math. Anal. 18(1987), 283-292.

[16] N. Kawano, E. Yanagida and S. Yotsutani, Structure theorems for positive radial solutions to $\Delta u+$ $K(|x|) u^{p}=0$ in $R^{n}$, Funkcialaj Ekvac. 36(1993), 557-579.

[17] R. W. Leggett and L. R. Williams, Multiple positive fixed points of nonlinear operators on ordered Banach spaces, Indiana Univ. Math. J. 28(1979), 673-688.

[18] Yansheng Liu, Boundary value problems for second order differential equations on unbounded domains in a Banach space, Appl. Math. Comput. 135(2003), 569-583.

[19] Yansheng Liu, Existence and unboundedness of positive solutions for singular boundary value problems on half-line,, Appl. Math. Comput. 144(2003), 543-556.

[20] R. Y. Ma and Y. N. Raffoul, Positive solutions of three-point nonlinear discrete second order boundary value problem, J. Difference Equations Applications 10(2004), 129-138.

[21] H. B. Thompson, Topological methods for some boundary value problems, Comput. Math. Appl. 42(2001), 487-495.

[22] Yu Tian, Dexiand Ma and Weigao Ge, Multiple positive solutions of four point boundary value problems for finite difference equations, J. Difference Equ. Appl. 12(2006), 57-68.

[23] Yu Tian and Weigao Ge, Multiple positive solutions of boundary value problems or second-order discrete equations on the Half-Line, J. Difference Equ. Appl. 12(2006), 191-208.

[24] P. J. Y. Wong and R. P. Agarwal, Eigenvalues of boundary value problems for higher order differential equations, Math. Prol. Eng. 2(1996), 401-434.

[25] M. Zima, On positive solutions of boundary value problems on the half-line, J. Math. Anal. Appl. 259(2001), 127-136.

School of Science, Beijing University of Posts and Telecommunications, Beijing 100876, P. R. China.

E-mail: tianyu2992@bit.edu.cn

Department of Applied Mathematics, Beijing Institute of Technology, Beijing 100081, P. R. China. 